\title{
Towards a construction of inclusive collision cross-sections in the massless Nelson model
}

\author{
Wojciech Dybalski* \\ Zentrum Mathematik, Technische Universität München, \\ D-85747 Garching, Germany \\ E-mail: dybalski@ma.tum.de
}

\begin{abstract}
The conventional approach to the infrared problem in perturbative quantum electrodynamics relies on the concept of inclusive collision cross-sections. A non-perturbative variant of this notion was introduced in algebraic quantum field theory. Relying on these insights, we take first steps towards a non-perturbative construction of inclusive collision cross-sections in the massless Nelson model. We show that our proposal is consistent with the standard scattering theory in the absence of the infrared problem and discuss its status in the infrared-singular case.
\end{abstract}

\section{Introduction}

The interpretation of physical states of quantum electrodynamics (QED) at asymptotic times is plagued by the computational and conceptual difficulties known as the infrared problem [4. On the perturbative side a partial solution was given in [29, 40] with the help of the concept of inclusive cross-sections. These crosssections incorporate all the outgoing photon configurations whose energy is below the sensitivity of the detector. An attempt to go beyond the inclusive cross-sections was made in [12,20,31, where a concrete expression for scattering states involving soft-photon clouds (i.e. infinite families of low energy photons) was proposed.

The two approaches mentioned above have their counterparts on the nonperturbative side: In the algebraic framework of local relativistic quantum field theory (QFT) a model independent construction of inclusive cross-sections, proposed in [7, 8] and developed further in [18, 19, 30, 34, 35], is a promising yet still incomplete programme. In the complementary setting of non-relativistic QED careful choices of soft-photon clouds resulted in a rigorous construction of scattering states for a class of simplified models of QED [10, 11, 15, 32, 33, including the Nelson model.

*Supported by the DFG grant SP181/25. 
In the present paper we take first steps towards a construction of inclusive cross-sections in the massless Nelson model along the lines set in algebraic QFT. Let us therefore recall the formulation of QED in this latter setting. As usually in relativistic quantum theory, one assumes the existence of the total energy and momentum operators $(H, P)$, acting on the physical Hilbert space $\mathcal{H}$, whose joint spectrum is contained in the closed forward lightcone. Moreover, $\mathcal{H}$ carries the Faraday tensor $F^{\mu \nu}$ and the conserved current $j^{\nu}$, defined as Wightman quantum fields (i.e. certain distributions taking values in unbounded operators on a domain in $\mathcal{H}$ [39]), and subject to the Maxwell equations. By smearing the distributions $j^{\nu}, F^{\mu \nu}$ with suitable test-functions and taking bounded functions of the resulting operators one obtains elements of the algebra of observables $\mathfrak{A} \subset B(\mathcal{H})$.

As shown in [6] using the Maxwell equations, the physical Hilbert space does not contain single-electron states. More precisely, the relativistic mass operator $\sqrt{H^{2}-P^{2}}$ has no eigenvectors whose electric charge is different from zero. Hence the electron is an infraparticle what precludes the conventional approach to scattering theory based on the LSZ asymptotic fields [5, 17, 27, 37]. An alternative approach, proposed in [7,8], aims at a direct construction of inclusive cross-sections, without recourse to asymptotic fields. As a first step one has to identify in the theoretical setting particle detectors of some finite energy sensitivity $\delta$. To this end one chooses an observable $B_{\delta} \in \mathfrak{A}$ which decreases energy by $\delta$ and is almost local in the sense of [2]. Next, one introduces the following sequences of observables

$$
C_{\delta, t}(\chi):=\mathrm{e}^{i H t}\left(\int_{\mathbb{R}^{3}} d x \chi(x / t) \mathrm{e}^{-i P x}\left(B_{\delta}^{*} B_{\delta}\right) \mathrm{e}^{i P x}\right) \mathrm{e}^{-i H t}
$$

which have the interpretation, as $t \rightarrow \infty$, of particle detectors sensitive to particles whose velocity is contained in the support of the function $\chi \in C_{0}^{\infty}\left(\mathbb{R}^{3}\right)$ and whose energy is larger than $\delta$ [2]. It was shown in [7] that in any local, relativistic QFT the sequences $\left\{C_{\delta, t}(\chi)\right\}_{t \in \mathbb{R}}$ have weak limit points $C_{\delta}^{+}$as $t \rightarrow \infty$ which are closable operators on a dense domain of states of bounded energy. The structure of these limit points was investigated in [34, 35], conditions for their non-triviality were found in [18] and a general scheme for construction of inclusive collision crosssections was proposed in [8]. So far this scheme was tested only in asymptotically complete theories of massive particles, where no infrared problems arise [38. More importantly, the problem of convergence of the sequences $\left\{C_{\delta, t}(\chi)\right\}_{t \in \mathbb{R}}$, which is essential for their interpretation as particle detectors, remains open to date in the presence of infraparticles. This problem is the main obstacle to the understanding of infraparticle scattering in the general framework of algebraic QFT. In order to shed some light on this issue, we reformulate the programme outlined in [8] in the setting of non-relativistic QED, where this and related questions appear to be more tractable.

Let us consider the massless, translationally invariant Nelson model given by 
the Hamiltonian and momentum operators

$$
\begin{aligned}
H & =\frac{p^{2}}{2 M}+\int_{\mathbb{R}^{3}} d q|q| a_{q}^{*} a_{q}+\lambda \int_{\mathbb{R}^{3}} d q \frac{\hat{\rho}(q)}{\sqrt{2|q|}}\left(\mathrm{e}^{-i q x} a_{q}^{*}+\mathrm{e}^{i q x} a_{q}\right), \\
P & =p+\int_{\mathbb{R}^{3}} d q q a_{q}^{*} a_{q},
\end{aligned}
$$

acting on the Hilbert space $\mathcal{H}=L^{2}\left(\mathbb{R}^{3}\right) \otimes \Gamma\left(L^{2}\left(\mathbb{R}^{3}\right)\right)$. Here the first factor is the space of states of the massive particle, which we call 'electron', whose position and momentum operators are denoted by $(x, p)$. The second factor is the bosonic Fock space containing states of scalar massless particles, which we call 'photons'. The corresponding creation and annihilation operators are denoted by $a_{q}^{*}, a_{q}$. The form factor $\hat{\rho} \in C_{0}^{\infty}\left(\mathbb{R}^{3}\right)$ is the Fourier transform of the charge density $\rho$ of the electron. If the total charge $(2 \pi)^{3 / 2} \hat{\rho}(0)$ and the coupling constant $\lambda$ are different from zero, then the Hilbert space does not contain states describing a single (dressed) massive particle. More precisely, the operator $H-E(P)$, where $\xi \rightarrow E(\xi)$ is the lower boundary of the joint spectrum of the family of commuting operators $(H, P)$, has no eigenvectors with eigenvalue zero [21,28]. Scattering states in this infraparticle situation were constructed in [32, 33] by a careful description of the soft-photon clouds accompanying the massive particle. The goal of the present work is complementary: We propose a strategy aiming at a direct construction of (inclusive) cross-sections, avoiding a consideration of scattering states. The first step is to identify suitable counterparts of the particle detectors (1.1) in the present concrete setting.

To construct a photon detector, we use the following approximants

$$
C_{g, t}(\chi):=\mathrm{e}^{i t H} \mathrm{~d} \Gamma(g \chi(y / t) g) \mathrm{e}^{-i t H},
$$

where $(y, k)$ are the photon position and momentum operators, $\chi, g \in C_{0}^{\infty}\left(\mathbb{R}^{3}\right)$ are real valued, the support of $g$ is isolated from zero, and we denote $g:=g(k)$. As $t \rightarrow \infty$, this observable has an interpretation of a detector sensitive to photons whose momentum belongs to the support of $g$ and whose direction of motion is restricted by the support of $\chi$ (cf. Proposition 3.2). $\left\{C_{g, t}(\chi)\right\}_{t \in \mathbb{R}}$ converges strongly to the limit $C_{g}^{+}(\chi)$ on all vectors from the relevant energy range. This fact, stated as Theorem 5.1 below, can be proven using methods developed in [14,23].

As an electron detector we propose the asymptotic velocity of the electron, approximated by

$$
C_{t}(f):=\mathrm{e}^{i t H} f(x / t) \mathrm{e}^{-i t H},
$$

where $f \in C_{0}^{\infty}\left(\mathbb{R}^{3}\right)$. At asymptotic times this observable is sensitive to electrons whose velocity belongs to the support of $f$ (cf. Proposition 3.1). In the infraparticle situation the convergence of $\left\{C_{t}(f)\right\}_{t \in \mathbb{R}}$ to a particle detector $C^{+}(f)$ is known only on the infraparticle scattering states constructed in [32, 33. The problem of convergence of this sequence of operators on all vectors from the relevant energy subspace does not seem to be amenable to existing methods. This important open problem will not be addressed in the present paper, apart from some remarks in Section 5 , 
Let us now outline the construction of inclusive cross-sections with the help of the particle detectors $C_{g}^{+}(\chi)$ and $C^{+}(f)$, which is the main subject of the present paper. To leave the problem of convergence of $\left\{C_{t}(f)\right\}_{t \in \mathbb{R}_{+}}$aside, let us introduce an infrared cut-off i.e. suppose that $q \rightarrow \hat{\rho}(q)$ vanishes in a neighbourhood of zero. Under this assumption it was shown in [23] that for a sufficiently small coupling constant $\lambda$ the model is asymptotically complete up to a certain energy value $\Sigma>\inf \sigma(H)$. Restricting attention to the corresponding subspace $\mathbf{1}_{(-\infty, \Sigma]}(H) \mathcal{H}$ of the Hilbert space, it is easy to prove the existence of the particle detectors $C_{g}^{+}(\chi)$ and $C^{+}(f)$. (See Propositions 3.1 and 3.2). Now let $S^{+} \subset \mathbb{R}^{3}$ be a compact subset of momenta of the electron. With the help of a sequence of functions $f_{n}(\nabla E(\cdot))$, tending pointwise to the characteristic function of $S^{+}$, we construct $C_{S^{+}}^{+}:=\mathrm{s}-\lim _{n} C^{+}\left(f_{n}\right)$. Similarly, for any compact subset $R^{+} \subset \mathbb{R}^{3} \backslash\{0\}$ of photon momenta, the corresponding detector $C_{R^{+}}^{+}$is constructed. Next, for any prescribed configuration of (hard) particles, given by mutually disjoint sets $S^{+}, R_{1}^{+}, \ldots, R_{n^{+}}^{+}$, we define the set of total energies of this configuration

$$
\Delta=\left\{E(\xi)+\left|q_{1}\right|+\cdots+\left|q_{n^{+}}\right| \mid \xi \in S^{+}, q_{1} \in R_{1}^{+}, \ldots, q_{n^{+}} \in R_{n^{+}}^{+}\right\} .
$$

Then $|\Delta|:=\sup \Delta-\inf \Delta$ gives the experimental uncertainty of the total energy of the system. The energies of hard photons should be larger than this uncertainty i.e. $|\Delta|<\inf \left\{|q| \mid q \in R_{j}^{+}, 1 \leq j \leq n^{+}\right\}$should hold. It is our main result that under this assumption the operator

$$
Q^{+}:=\mathbf{1}_{\Delta}(H) C_{R_{1}^{+}}^{+} \ldots C_{R_{n^{+}}^{+}}^{+} C_{S^{+}}^{+}
$$

is an orthogonal projection thus it has a clear quantum mechanical interpretation in terms of particle measurements. Every non-zero vector from the range of this projection describes the prescribed configuration of hard particles and some unspecified configuration of soft photons whose total energy is less than $|\Delta|$. (See Theorem [4.1). By reversing the time direction, the above construction can be repeated for an incoming configuration of hard particles $S^{-}, R_{1}^{-}, \ldots, R_{n^{-}}^{-}$resulting in a projection $Q^{-}$. The (inclusive) cross-section for the collision process $S^{-}, R_{1}^{-}, \ldots, R_{n^{-}}^{-} \rightarrow S^{+}, R_{1}^{+}, \ldots, R_{n^{+}}^{+}$is proportional to the transition probability

$$
\operatorname{Pr}=\left|\left(\frac{Q^{-} \Psi^{-}}{\left\|Q^{-} \Psi^{-}\right\|}, \frac{Q^{+} \Psi^{+}}{\left\|Q^{+} \Psi^{+}\right\|}\right)\right|^{2},
$$

where $\Psi^{ \pm} \in \mathbf{1}_{(-\infty, \Sigma]}(H) \mathcal{H}$ are chosen so that $Q^{ \pm} \Psi^{ \pm} \neq 0$. The physical situation dictates the choice $\Psi^{+}=Q^{-} \Psi^{-}$. Then, exploiting the fact that $Q^{ \pm}$are orthogonal projections, we obtain

$$
\operatorname{Pr}=\left(\frac{Q^{-} \Psi^{-}}{\left\|Q^{-} \Psi^{-}\right\|}, Q^{+} \frac{Q^{-} \Psi^{-}}{\left\|Q^{-} \Psi^{-}\right\|}\right) .
$$

The remaining dependence of this quantity on the vector $\Psi^{-}$reflects the ambiguities inherent in the experimental procedure of the initial state preparation. 
While the technical aspects of the above construction still rely on the standard scattering theory, we conjecture that the operators $Q^{ \pm}$also exist and are orthogonal projections in the presence of the infrared problem. In this general context they should provide a natural language for a description of collision processes, which has a counterpart in the relativistic setting of algebraic QFT [8].

Our paper is organized as follows: In Section 2 we recall some known facts about the Nelson model which are relevant to the present investigation. In Section 3 the convergence of the particle detector approximants (1.4) and (1.5) is established on the subspace of scattering states. The proofs of these results, which exploit methods presented in [23], are given in the Appendices. In Section 4 we prove that the operators $Q^{+}$, given by (1.7), are orthogonal projections on the subspace of scattering states. In Section 5 we discuss the feasibility of our construction in the presence of the infrared problem. In particular, we point out that the convergence of photon detectors can be established in this situation.

\section{Preliminaries}

In this section, which serves mostly to introduce notation, we provide a survey of some known facts about the Nelson model, which will be needed in the sequel.

Let $\mathfrak{h}$ be a Hilbert space and let $\Gamma(\mathfrak{h}):=\oplus_{n \geq 0} \otimes_{s}^{n} \mathfrak{h}$ be the symmetric Fock space over $\mathfrak{h}$. We denote by $a^{*}(h)$ and $a(h), h \in \mathfrak{h}$, the creation and annihilation operators and set $\phi(h)=a^{*}(h)+a(h)$. For any self-adjoint operator $b$, acting on (a dense domain in) $\mathfrak{h}$, we denote by $\mathrm{d} \Gamma(b)$ a self-adjoint operator, acting on (a dense domain in) $\Gamma(\mathfrak{h})$, defined by

$$
\left.\mathrm{d} \Gamma(b)\right|_{\otimes_{s}^{n} \mathfrak{h}}=\sum_{i=1}^{n}(\underbrace{I \otimes \cdots \otimes I}_{i-1} \otimes b \otimes I \otimes \cdots I) .
$$

For a more detailed exposition of the Fock space combinatorics we refer e.g. to [23].

Let $\mathcal{H}_{\mathrm{el}}=L^{2}\left(\mathbb{R}^{3}\right)$ be the Hilbert space of the (bare) electron, whose position and momentum operators are denoted by $(x, p)$. Let $\mathfrak{h}=L^{2}\left(\mathbb{R}^{3}\right)$ be the Hilbert space of a single photon, whose position and momentum operators are denoted by $(y, k)$. The normalized elements of $\mathcal{H}_{\mathrm{el}}$ (resp. $\left.\mathfrak{h}\right)$ are electron (resp. photon) wave-functions in momentum space. On the full Hilbert space $\mathcal{H}=\mathcal{H}_{\mathrm{el}} \otimes \Gamma(\mathfrak{h})$ we define the Hamiltonian and momentum operators of the Nelson model

$$
\begin{aligned}
H & =\Omega(p)+H_{\mathrm{ph}}+\phi\left(G_{x}\right), \\
P & =p+P_{\mathrm{ph}} .
\end{aligned}
$$

Here $\Omega(p)=\frac{p^{2}}{2 M} \otimes I, H_{\mathrm{ph}}=I \otimes \mathrm{d} \Gamma(\omega)$, where $\omega(q)=|q|$ and $\omega:=\omega(k), P_{\mathrm{ph}}=$ $I \otimes \mathrm{d} \Gamma(k), G_{x}(q)=\kappa(q) \mathrm{e}^{-i q x}$, where $\kappa(q)=\lambda \frac{\hat{\rho}(q)}{\sqrt{2 \omega(q)}}$ and $\hat{\rho} \in C_{0}^{\infty}\left(\mathbb{R}^{3}\right)$ is a positive function. It is a consequence of the Kato-Rellich theorem that $H$ is self-adjoint on the domain of self-adjointness of $H_{0}:=\Omega(p)+H_{\mathrm{ph}}$ and its spectrum $\sigma(H)$ is bounded from below. 
The operators $(H, P)$, given by (2.2), (2.3), form a family of four commuting self-adjoint operators on a domain in $\mathcal{H}$. The lower boundary of their joint spectrum is denoted by $\xi \rightarrow E(\xi)$. We recall the following facts:

Lemma 2.1. There exists $\Sigma>\inf \sigma(H)$ and $\lambda>0$ s.t. for $E(\xi)<\Sigma$ and some $\varepsilon>0$

(a) $\xi \rightarrow E(\xi)$ is real analytic,

(b) $\xi \rightarrow \nabla E(\xi)$ is invertible,

(c) $|\nabla E(\xi)|<1$,

(d) ||$|\nabla \Omega(p)| \mathbf{1}_{(-\infty, \Sigma+\varepsilon]}(H) \|<1$.

Parts (a)-(c) of this lemma follow from [1]. Part (d) is a consequence of Lemma 11 of [23]. We assume in the sequel that $\Sigma$ and $\lambda$ are chosen as in the above lemma.

Next, we define the subspace of dressed single-particle states

$$
\mathcal{H}_{\text {des }}=\mathbf{1}_{(-\infty, \Sigma]}(H) \mathbf{1}_{\{0\}}(H-E(P)) \mathcal{H} \text {. }
$$

In Sections 3 and 4 we assume that $\mathcal{H}_{\text {des }} \neq\{0\}$. This holds e.g. in the presence of an infrared cut-off, i.e. if $q \rightarrow \hat{\rho}(q)$ vanishes in some neighbourhood of zero [21,23], and is expected to hold whenever the massive particle is neutral i.e. if $\hat{\rho}(0)=0$. On the other hand, $\mathcal{H}_{\text {des }}=\{0\}$ if $\lambda \neq 0$ and the massive particle is charged i.e. $\hat{\rho}(0)>0[21,28]$. We hope that our construction of inclusive cross-sections can be extended to this general situation. We discuss some first steps in Section 5 .

Proceeding to the construction of asymptotic creation and annihilation operators of massless particles, we introduce the spaces

$$
\begin{aligned}
L_{\omega}^{2}\left(\mathbb{R}^{3}\right) & =\left\{\left.h \in L^{2}\left(\mathbb{R}^{3}\right)\left|\|h\|_{\omega}^{2}:=\int_{\mathbb{R}^{3}} d q\right| h(q)\right|^{2}\left(1+\omega(q)^{-1}\right)<\infty\right\}, \\
L^{2, \omega}\left(\mathbb{R}^{3}\right) & =\left\{h \in L_{\omega}^{2}\left(\mathbb{R}^{3}\right) \mid \omega h \in L_{\omega}^{2}\left(\mathbb{R}^{3}\right)\right\} .
\end{aligned}
$$

We recall the following results:

Lemma 2.2. (a) For every $n \in \mathbb{N}$ the operator $H_{\mathrm{ph}}^{n}(H+i)^{-n}$ is bounded. (b) For every $n \in \mathbb{N}$ there is a constant $C_{n}$, s.t. for all $h_{1}, \ldots, h_{n} \in L_{\omega}^{2}\left(\mathbb{R}^{3}\right)$

$$
\left\|a^{\#}\left(h_{1}\right) \ldots a^{\#}\left(h_{n}\right)\left(H_{\mathrm{ph}}+1\right)^{-n / 2}\right\| \leq C_{n} \prod_{j=1}^{N}\left\|h_{j}\right\|_{\omega}
$$

where $a^{\#}(\cdot)$ stands for a creation or annihilation operator.

Part (a) of this lemma is an adaptation of an argument from [22] to the case of the Nelson model, part (b) is standard. In view of the above lemma, we can define the asymptotic creation operator

$$
a_{+}^{*}(h) \Psi:=\mathrm{s}^{-} \lim _{t \rightarrow \infty} \mathrm{e}^{i t H} a^{*}\left(h_{t}\right) \mathrm{e}^{-i t H} \Psi
$$


where $h_{t}=\mathrm{e}^{-i \omega t} h$ and $\Psi$ is any vector from the domain of $(c+H)^{\frac{1}{2}}$ for which the limit exists. (Here $c \geq 0$ is s.t. $\sigma(H)+c \subset(0, \infty)$ ). The following fact, which is a result from [24] adapted to the case of the Nelson model, concerns the properties of these operators:

Lemma 2.3. Choose $\Sigma>\inf \sigma(H)$ and $\lambda>0$ s.t. $\left\||\nabla \Omega(p)| \mathbf{1}_{(-\infty, \Sigma+\varepsilon]}(H)\right\|<1$ for some $\varepsilon>0$. (Cf. Lemma 2.1). Then, for any $h_{1}, \ldots, h_{n} \in L^{2, \omega}\left(\mathbb{R}^{3}\right)$ and $\Psi \in \mathbf{1}_{(-\infty, \Sigma]}(H) \mathcal{H}$ the following limit

$$
\mathrm{s}-\lim _{t \rightarrow \infty} \mathrm{e}^{i t H} a^{\#}\left(h_{1, t}\right) \ldots a^{\#}\left(h_{n, t}\right) \mathrm{e}^{-i t H} \Psi
$$

exists and equals

$$
a_{+}^{\#}\left(h_{1}\right) \ldots a_{+}^{\#}\left(h_{n}\right) \Psi
$$

Let us now prove the following lemma, which is a sharpened variant of Theorem 4 (iv) of [22]:

Lemma 2.4. Let $\Psi \in \operatorname{Ran}_{\left[E_{1}, E_{2}\right]}(H)$, where $E_{1} \leq E_{2} \leq \Sigma$. Let $h_{1}, \ldots, h_{n} \in$ $L^{2, \omega}\left(\mathbb{R}^{3}\right)$ be functions of compact support (in the sense of distributions), $m_{j}:=$ $\inf \left\{\omega(q) \mid q \in \operatorname{supp} h_{j}\right\}$ and $M_{j}:=\sup \left\{\omega(q) \mid q \in \operatorname{supp} h_{j}\right\}$. Then the vector

$$
\Psi_{n}:=a_{+}^{*}\left(h_{n}\right) \ldots a_{+}^{*}\left(h_{1}\right) \Psi
$$

belongs to $\operatorname{Ran} \mathbf{1}_{\left[E_{1}+m_{1}+\cdots+m_{n}, E_{2}+M_{1}+\cdots+M_{n}\right]}(H)$.

Proof. We proceed by induction. For $n=0$ the statement is trivially true. Suppose it holds for $n-1$ creation operators. We choose $\hat{f} \in C_{0}^{\infty}(\mathbb{R})$ s.t. $\hat{f}(\omega(\cdot))=$ 1 on $\operatorname{supp} h_{n}$ and vanishes on a slightly larger set. We write

$$
\begin{aligned}
& a_{+}^{*}\left(h_{n}\right) \Psi_{n-1}=a_{+}^{*}\left(\hat{f}(\omega) h_{n}\right) \Psi_{n-1} \\
& =\mathrm{s}-\lim _{t \rightarrow \infty} \frac{1}{\sqrt{2 \pi}} \int d s f(s) \mathrm{e}^{i H t} a^{*}\left(\mathrm{e}^{-i \omega t} \mathrm{e}^{i \omega s} h_{n}\right) \mathrm{e}^{-i H t} \Psi_{n-1} \\
& =\mathrm{s}-\lim _{t \rightarrow \infty} \frac{1}{\sqrt{2 \pi}} \int d s f(s) \mathrm{e}^{i H s}\left(\mathrm{e}^{i H t} a^{*}\left(\mathrm{e}^{-i \omega t} h_{n}\right) \mathrm{e}^{-i H t}\right) \mathrm{e}^{-i H s} \Psi_{n-1},
\end{aligned}
$$

where in the last step we made use of the dominated convergence theorem for Bochner integrable functions to enter with the limit under the integral sign and make a change of variables. (Norm continuity of the integrand follows from Lemma 2.2). Next, we recall that for any $A \in B(\mathcal{H})$ and compact set $\Delta \subset \mathbb{R}$ the operator $A(f):=\int d s f(s) \mathrm{e}^{i H s} A \mathrm{e}^{-i H s}$ satisfies [3]

$$
A(f) \mathbf{1}_{\Delta}(H) \mathcal{H} \subset \mathbf{1}_{\{\Delta+\operatorname{supp} \tilde{f}\}}(H) \mathcal{H} .
$$

Each operator $A:=\mathrm{e}^{i H t} a^{*}\left(\mathrm{e}^{-i \omega t} h_{n}\right) \mathrm{e}^{-i H t} \mathbf{1}_{\Delta_{n-1}}(H)$, where $\Delta_{n-1}=\left[E_{1}+m_{1}+\cdots+\right.$ $\left.m_{n-1}, E_{2}+M_{1}+\cdots+M_{n-1}\right]$, belongs to $B(\mathcal{H})$ by Lemma 2.2. Making use of the induction hypothesis and of formula (2.13), we complete the proof.

Let $L_{\mathrm{c}}^{2, \omega}\left(\mathbb{R}^{3}\right)$ be the subspace of functions from $L^{2, \omega}\left(\mathbb{R}^{3}\right)$ which are compactly supported (in the sense of distributions). We define the following space

$\mathcal{H}_{0}^{+}=\operatorname{Span}\left\{a_{+}^{*}\left(h_{1}\right) \ldots a_{+}^{*}\left(h_{n}\right) \Psi \mid \Psi \in \mathcal{H}_{\mathrm{des}}, h_{j} \in L_{\mathrm{c}}^{2, \omega}\left(\mathbb{R}^{3}\right), 1 \leq j \leq n, n \in \mathbb{N}\right\}$

as well as its norm closure $\mathcal{H}^{+}=\left(\mathcal{H}_{0}^{+}\right)^{\mathrm{cl}}$. 


\section{Particle detectors}

In this section we collect the relevant properties of the electron detectors $C^{+}(f)$ and the photon detectors $C_{g}^{+}(\chi)$, defined as limits of the respective sequences

$$
\begin{aligned}
C_{t}(f) & :=\mathrm{e}^{i t H} f(x / t) \mathrm{e}^{-i t H}, \\
C_{g, t}(\chi) & :=\mathrm{e}^{i t H} \mathrm{~d} \Gamma(g \chi(y / t) g) \mathrm{e}^{-i t H},
\end{aligned}
$$

as $t \rightarrow \infty$. Here $f \in C_{0}^{\infty}\left(\mathbb{R}^{3}\right), \chi, g \in C_{0}^{\infty}\left(\mathbb{R}^{3}\right)_{\mathbb{R}}$, the support of $g$ does not contain zero and we wrote $g:=g(k)$ in (3.2) above. In particular, we show that these limits exist on vectors from $\mathcal{H}_{0}^{+}$.

The following proposition describes the action of the electron detector on scattering states. Although this result may be known (cf. Remark $2 \mathrm{i}$ in [33]), we have not found a proof in the literature. Thus we include the straightforward argument in Appendix $\mathrm{A}$.

Proposition 3.1. Let $h_{1}, \ldots, h_{n} \in L_{\mathrm{c}}^{2, \omega}\left(\mathbb{R}^{3}\right), f \in C_{0}^{\infty}\left(\mathbb{R}^{3}\right)$ and $\Psi \in \mathcal{H}_{\mathrm{des}}$. Then

$$
\text { s- } \lim _{t \rightarrow \infty} \mathrm{e}^{i H t} f(x / t) \mathrm{e}^{-i H t} a_{+}^{*}\left(h_{1}\right) \ldots a_{+}^{*}\left(h_{n}\right) \Psi=a_{+}^{*}\left(h_{1}\right) \ldots a_{+}^{*}\left(h_{n}\right) f(\nabla E(P)) \Psi .
$$

The action of the photon detector on scattering states is described in the following proposition, proven in Appendix B.

Proposition 3.2. Let $\varepsilon>0$ be s.t. $|\nabla E(\xi)|<1-\varepsilon$ for $E(\xi) \leq \Sigma$. (Cf. Lemma[2.1). Choose $\chi \in C_{0}^{\infty}\left(\mathbb{R}^{3}\right)_{\mathbb{R}}$, s.t. $\operatorname{supp} \chi \subset\left\{v \in \mathbb{R}^{3}|||v|-1 \mid \leq \varepsilon / 4\right\}$ and $\chi(v)=1$ if $|v|=1$. Let $h_{1}, \ldots, h_{n} \in L_{\mathrm{c}}^{2, \omega}\left(\mathbb{R}^{3}\right)$ and $\Psi \in \mathcal{H}_{\mathrm{des}}$. Then

$$
\begin{aligned}
\mathrm{s}-\lim _{t \rightarrow \infty} \mathrm{e}^{i H t} \mathrm{~d} \Gamma(g \chi(y / t) g) \mathrm{e}^{-i H t} a_{+}^{*}\left(h_{1}\right) \ldots a_{+}^{*}\left(h_{n}\right) \Psi \\
=\sum_{j=1}^{n} a_{+}^{*}\left(h_{1}\right) \ldots a_{+}^{*}\left(g^{2} h_{j}\right) \ldots a_{+}^{*}\left(h_{n}\right) \Psi
\end{aligned}
$$

for $g \in C_{0}^{\infty}\left(\mathbb{R}^{3}\right)_{\mathbb{R}}$ vanishing in a neighbourhood of zero.

The above results justify the physical interpretation of $C^{+}(f), C_{g}^{+}(\chi)$, as particle detectors sensitive to particles whose momenta are specified by the supports of the functions $f$ and $g$. For the construction of collision cross-sections it is essential that these operators are invariant under time translations. While it is evident on $\mathcal{H}_{0}^{+}$by the above two propositions, it can easily be shown in more generality.

Proposition 3.3. Let $\Sigma^{\prime}>0$ be arbitrary and let $\Psi_{1}, \Psi_{2} \in \mathbf{1}_{\left(-\infty, \Sigma^{\prime}\right]}(H) \mathcal{H}$ be s.t. the limits

$$
\begin{aligned}
& \mathrm{e}^{i s H} C^{+}(f) \mathrm{e}^{-i s H} \Psi_{1}:=\quad \mathrm{s}-\lim _{t \rightarrow \infty} \mathrm{e}^{i(t+s) H} f(x / t) \mathrm{e}^{-i(t+s) H} \Psi_{1}, \\
& \mathrm{e}^{i s H} C_{g}^{+}(\chi) \mathrm{e}^{-i s H} \Psi_{2}:=\mathrm{s}^{-} \lim _{t \rightarrow \infty} \mathrm{e}^{i(t+s) H} \mathrm{~d} \Gamma(g \chi(y / t) g) \mathrm{e}^{-i(t+s) H} \Psi_{2}
\end{aligned}
$$

exist for $s=0$. Then the limits exist for all $s \in \mathbb{R}$ and $\mathrm{e}^{i s H} C^{+}(f) \mathrm{e}^{-i s H} \Psi_{1}=$ $C^{+}(f) \Psi_{1}, \mathrm{e}^{i s H} C_{g}^{+}(\chi) \mathrm{e}^{-i s H} \Psi_{2}=C_{g}^{+}(\chi) \Psi_{2}$.

The proof of this proposition, which partly comes from [23], is given in Appendix C, 


\section{Inclusive collision cross-sections}

In this section we give a detailed construction of the operators $Q^{+}$, defined precisely in (4.4) below, which we used to define transition probabilities of inclusive collision processes in (1.9). In particular, we show that these operators are orthogonal projections on $\mathcal{H}^{+}$, what validates their interpretation as coincidence arrangements of particle detectors.

Let $S^{+} \subset \mathbb{R}^{3}$ be a compact subset of electron momenta s.t. $E(\xi) \leq \Sigma$ for $\xi \in S^{+}$. Making use of Lemma 2.1 (b), we choose a family of functions $f_{\varepsilon} \in C_{0}^{\infty}\left(\mathbb{R}^{3}\right)$ s.t. $\xi \rightarrow f_{\varepsilon}(\nabla E(\xi))$ approximates pointwise the characteristic function $\mathbf{1}_{S^{+}}(\cdot)$ of the set $S^{+}$as $\varepsilon \rightarrow 0$. Exploiting Proposition 3.1 and Lemma 2.2, we can meaningfully define for any $h_{1}, \ldots, h_{n} \in L_{\mathrm{c}}^{2, \omega}\left(\mathbb{R}^{3}\right)$ and $\Psi \in \mathcal{H}_{\text {des }}$

$$
\begin{aligned}
C_{S^{+}}^{+} a_{+}^{*}\left(h_{1}\right) \ldots a_{+}^{*}\left(h_{n}\right) \Psi & :=\mathrm{s}^{-} \lim _{\varepsilon \rightarrow 0} \mathrm{~s}^{-} \lim _{t \rightarrow \infty} \mathrm{e}^{i H t} f_{\varepsilon}(x / t) \mathrm{e}^{-i H t} a_{+}^{*}\left(h_{1}\right) \ldots a_{+}^{*}\left(h_{n}\right) \Psi \\
& =a_{+}^{*}\left(h_{1}\right) \ldots a_{+}^{*}\left(h_{n}\right) \mathbf{1}_{S^{+}}(P) \Psi .
\end{aligned}
$$

Clearly, $C_{S^{+}}^{+}$extends to an orthonormal projection on $\mathcal{H}^{+}$.

Now let $R^{+} \subset \mathbb{R}^{3}$ be a compact subset of photon momenta s.t. $R^{+} \cap\{0\}=\emptyset$. Let $q \rightarrow g_{\varepsilon}(q)$ be a family of positive functions from $C_{0}^{\infty}\left(\mathbb{R}^{3}\right)$, vanishing in some neighbourhood of zero, which tends pointwise to the characteristic function $\mathbf{1}_{R^{+}}(\cdot)$ of $R^{+}$and let $\chi$ be chosen as in Proposition 3.2. Making use of Proposition 3.2 and Lemma 2.2 , we define

$$
\begin{array}{rl}
C_{R^{+}}^{+} a_{+}^{*}\left(h_{1}\right) \ldots a_{+}^{*}\left(h_{n}\right) \Psi & \\
:=\mathrm{s}-\lim _{\varepsilon \rightarrow 0} \mathrm{~s}^{-} \lim _{t \rightarrow \infty} \mathrm{e}^{i H t} & \mathrm{~d} \Gamma\left(g_{\varepsilon} \chi(y / t) g_{\varepsilon}\right) \mathrm{e}^{-i H t} a_{+}^{*}\left(h_{1}\right) \ldots a_{+}^{*}\left(h_{n}\right) \Psi \\
& =\sum_{j=1}^{n} a_{+}^{*}\left(h_{1}\right) \ldots a_{+}^{*}\left(\mathbf{1}_{R^{+}} h_{j}\right) \ldots a_{+}^{*}\left(h_{n}\right) \Psi .
\end{array}
$$

$C_{R^{+}}^{+}$is an unbounded operator. It is defined on $\mathcal{H}_{0}^{+} \subset \mathcal{H}^{+}$and leaves this subspace invariant.

Next, given a collection of sets $S^{+}, R_{1}^{+}, \ldots, R_{n}^{+}$, we define the set of total energies of the corresponding particle configuration

$$
\Delta=\left\{E(\xi)+\omega\left(q_{1}\right)+\cdots+\omega\left(q_{n}\right) \mid \xi \in S^{+}, q_{j} \in R_{j}^{+}, 1 \leq j \leq n\right\} .
$$

Then $|\Delta|:=\sup \Delta-\inf \Delta$ is the accuracy with which the total energy of this particle configuration is known. The sets $R_{1}^{+}, \ldots, R_{n}^{+}$should describe 'hard photons', whose energies are larger than $|\Delta|$. If this condition is met, then the corresponding operator $Q^{+}$, given by (4.4) below, is an orthogonal projection, what validates its interpretation as a quantum mechanical measurement. This is the content of the following theorem, which is our main result.

Theorem 4.1. Let $S^{+}, R_{1}^{+}, \ldots, R_{n}^{+}$be compact subsets of $\mathbb{R}^{3}$ s.t. $R_{i}^{+} \cap R_{j}^{+}=\emptyset$ for $i \neq j$ and $E(\xi) \leq \Sigma$ for $\xi \in S^{+}$. Let $\Delta$ be given by (4.3). If $|\Delta|<\inf \{\omega(q) \mid q \in$ $\left.R_{j}^{+}, 1 \leq j \leq n\right\}$, then the operator

$$
Q^{+}:=\mathbf{1}_{\Delta}(H) C_{R_{1}^{+}}^{+} \ldots C_{R_{n}^{+}}^{+} C_{S^{+}}^{+}
$$


is an orthogonal projection on $\mathcal{H}^{+}$. Moreover, $\operatorname{Ran} Q^{+}$is spanned by vectors of the form

$$
a_{+}^{*}\left(h_{1}\right) \ldots a_{+}^{*}\left(h_{n}\right) a_{+}^{*}\left(h_{n+1}^{\prime}\right) \ldots a_{+}^{*}\left(h_{m}^{\prime}\right) \Psi
$$

where $m \geq n, \Psi \in \mathbf{1}_{S^{+}}(P) \mathcal{H}_{\mathrm{des}}, h_{i}, h_{j}^{\prime} \in L_{\mathrm{c}}^{2, \omega}\left(\mathbb{R}^{3}\right), \operatorname{supp} h_{i} \subset R_{i}^{+}, \operatorname{supp} h_{j}^{\prime} \subset\{q \in$ $\left.\mathbb{R}^{3}|| q \mid \leq \delta_{j}\right\}, \delta_{n+1}+\cdots+\delta_{m} \leq|\Delta|$.

Proof. The subspace $\mathcal{H}_{0}^{+}$is spanned by vectors of the form

$$
\Psi^{+}=a_{+}^{*}\left(h_{1}\right) \ldots a_{+}^{*}\left(h_{m}\right) \Psi
$$

where $h_{i} \in L_{\mathrm{c}}^{2, \omega}\left(\mathbb{R}^{3}\right), 1 \leq i \leq m$ and $\Psi \in \mathcal{H}_{\text {des }}$. It is clear from Proposition 3.2 that $Q^{+} \Psi=0$ for $m<n$. For $m \geq n$ the expression $Q^{+} \Psi^{+}$is a sum of terms of the form

$$
\Psi_{1}^{+}:=\mathbf{1}_{\Delta}(H) a_{+}^{*}\left(\mathbf{1}_{R_{i_{1}}^{+}} h_{1}\right) \ldots a_{+}^{*}\left(\mathbf{1}_{R_{i_{n}}^{+}} h_{n}\right) a_{+}^{*}\left(h_{n+1}\right) \ldots a_{+}^{*}\left(h_{m}\right) \mathbf{1}_{S^{+}}(P) \Psi .
$$

We decompose $h_{n+i}=h_{n+i}^{\prime}+h_{n+i}^{\prime \prime}$, where $\operatorname{supp} h_{n+i}^{\prime \prime} \subset\left\{k \in \mathbb{R}^{3}|| k|\geq| \Delta \mid\right\}$, $\operatorname{supp} h_{n+i}^{\prime} \subset\left\{k \in \mathbb{R}^{3}|| k|<| \Delta \mid\right\}$ and $1 \leq i \leq(m-n)$. Then, $Q^{+} \Psi^{+}$is a sum of terms of the form

$$
\begin{aligned}
\Psi_{2}^{+}:=\mathbf{1}_{\Delta}(H) a_{+}^{*}\left(\mathbf{1}_{R_{i_{1}}} h_{1}\right) \ldots & a_{+}^{*}\left(\mathbf{1}_{R_{i_{n}}^{+}} h_{n}\right) a_{+}^{*}\left(h_{n+1}^{\prime \prime}\right) \ldots a_{+}\left(h_{n+j}^{\prime \prime}\right) \\
& \cdot a_{+}^{*}\left(h_{n+j+1}^{\prime}\right) \ldots a_{+}^{*}\left(h_{m}^{\prime}\right) \mathbf{1}_{S^{+}}(P) \Psi .
\end{aligned}
$$

We will show that terms for which $j \geq 1$ are zero. First, we note that

$$
E_{\max }:=\sup \Delta=\sup \left\{E(\xi) \mid \xi \in S^{+}\right\}+\sum_{j=1}^{n} \sup \left\{\omega(q) \mid q \in R_{j}^{+}\right\} .
$$

Next, we obtain from Lemma 2.4 that $\Psi_{2}^{+} \in \operatorname{Ran} \mathbf{1}_{\left[E_{\min }, \infty\right)}(H)$, where $E_{\text {min }}$ satisfies

$$
E_{\min } \geq \inf \left\{E(\xi) \mid \xi \in S^{+}\right\}+\sum_{j=1}^{n} \inf \left\{\omega(q) \mid q \in R_{j}^{+}\right\}+|\Delta| .
$$

Hence $E_{\min }-E_{\max } \geq 0$. Since $\Psi_{2}^{+}$is not an eigenvector of $H, \Psi_{2}^{+}=0$ for $j \geq 1$. We conclude that $Q^{+} \Psi^{+}$is a sum of terms of the form

$$
\Psi_{2}^{+}:=\mathbf{1}_{\Delta}(H) a_{+}^{*}\left(\mathbf{1}_{R_{i_{1}}^{+}} h_{1}\right) \ldots a_{+}^{*}\left(\mathbf{1}_{R_{i_{n}}^{+}} h_{n}\right) a_{+}^{*}\left(h_{n+1}^{\prime}\right) \ldots a_{+}^{*}\left(h_{m}^{\prime}\right) \mathbf{1}_{S^{+}}(P) \Psi .
$$

Since $C_{R_{i}^{+}}^{+}, C_{S^{+}}^{+}$commute with $H$ by Proposition $3.3, \mathbf{1}_{R_{i}^{+}} h_{j}^{\prime}=0$ by assumption and $\mathbf{1}_{R_{i^{\prime}}^{+}} \mathbf{1}_{R_{j^{\prime}}^{+}}=0$ for $i^{\prime} \neq j^{\prime}$, it is evident that $Q^{+} Q^{+} \Psi^{+}=Q^{+} \Psi^{+}$.

To prove the second part of the theorem, it suffices to justify the support property of the functions $h_{j}^{\prime}$. It follows from the above discussion that vectors of the form (4.11) span Ran $Q^{+}$. Let us fix $\varepsilon>0$ and let $l=(m-n)$ be the number of soft photons in $\Psi_{2}^{+}$. It suffices to consider the case $l>0$. We define $\chi_{j}$ to 
be the characteristic function of the set $\left\{q \in \mathbb{R}^{3}\left|(j-1) \frac{\varepsilon}{l} \leq\right| q \mid \leq j \frac{\varepsilon}{l}\right\}$, where $j \in\left\{1, \ldots,\left[l \frac{|\Delta|}{\varepsilon}\right]+1\right\}$. Then $h_{n+i}^{\prime}(q)=\sum_{j_{n+i}} \chi_{j_{n+i}}(q) h_{n+i}^{\prime}(q)$. Substituting this decomposition to (4.11), we obtain that $\Psi_{2}^{+}$is a sum of terms of the form

$$
\begin{aligned}
\Psi_{3}^{+}:=\mathbf{1}_{\Delta}(H) a_{+}^{*}\left(\mathbf{1}_{R_{i_{1}}^{+}} h_{1}\right) & \ldots a_{+}^{*}\left(\mathbf{1}_{R_{i_{n}}^{+}} h_{n}\right) \\
& \cdot a_{+}^{*}\left(\chi_{j_{n+1}} h_{n+1}^{\prime}\right) \ldots a_{+}^{*}\left(\chi_{j_{m}} h_{m}^{\prime}\right) \mathbf{1}_{S^{+}}(P) \Psi .
\end{aligned}
$$

If the above vector is non-zero, then Lemma 2.4 gives

$$
\left(j_{n+1}-1\right) \frac{\varepsilon}{l}+\cdots+\left(j_{m}-1\right) \frac{\varepsilon}{l} \leq|\Delta| .
$$

Now let $\delta_{n+i}:=\sup \left\{|q| \mid q \in \operatorname{supp} \chi_{j_{n+i}} h_{n+i}^{\prime}\right\}$. Since $\delta_{n+i} \leq j_{n+i} \frac{\varepsilon}{l}$, it follows from the above relation that $\delta_{n+1}+\cdots+\delta_{m} \leq|\Delta|+\varepsilon$. By choosing $\hat{f}_{n} \in S(\mathbb{R})$ s.t. s- $\lim _{n \rightarrow \infty} \hat{f}_{n}(H)=\mathbf{1}_{\Delta}(H)$, writing $\hat{f}_{n}(H)=\frac{1}{\sqrt{2 \pi}} \int d t \mathrm{e}^{i H t} f_{n}(t)$ and making use of the fact that $\mathrm{e}^{i H t} a_{+}^{*}(h) \mathrm{e}^{-i H t}=a_{+}^{*}\left(\mathrm{e}^{i \omega t} h\right)$, we obtain the following: For any $\varepsilon>0$, $Q^{+} \Psi^{+}$belongs to the subspace $\mathcal{H}_{\varepsilon}$ spanned by vectors of the form

$$
a_{+}^{*}\left(h_{1}\right) \ldots a_{+}^{*}\left(h_{n}\right) a_{+}^{*}\left(h_{n+1}^{\prime}\right) \ldots a_{+}^{*}\left(h_{m}^{\prime}\right) \Psi,
$$

where $m \geq n, \Psi \in \mathbf{1}_{S^{+}}(P) \mathcal{H}_{\mathrm{des}}, h_{i}, h_{j}^{\prime} \in L_{\mathrm{c}}^{2, \omega}\left(\mathbb{R}^{3}\right), \operatorname{supp} h_{i} \subset R_{i}^{+}, \operatorname{supp} h_{j}^{\prime} \subset\{k \in$ $\left.\mathbb{R}^{3}|| k \mid \leq \delta_{j}\right\}, \delta_{n+1}+\cdots+\delta_{m} \leq|\Delta|+\varepsilon$. By identifying $\mathcal{H}_{\varepsilon}$ with a subspace of $\Gamma\left(L^{2}\left(\left\{k \in \mathbb{R}^{3}|| k|\geq| \Delta \mid\right\}\right)\right) \otimes \Gamma\left(L^{2}\left(\left\{k \in \mathbb{R}^{3}|| k|\leq| \Delta \mid\right\}\right)\right) \otimes \mathcal{H}_{\text {des }}$ and noting that

$$
\mathrm{s}-\lim _{\varepsilon \rightarrow 0} I \otimes \mathbf{1}_{[0,|\Delta|+\varepsilon]}\left(H_{\mathrm{ph}}\right) \otimes I=I \otimes \mathbf{1}_{[0,|\Delta|]}\left(H_{\mathrm{ph}}\right) \otimes I,
$$

we obtain that $\bigcap_{\varepsilon>0} \mathcal{H}_{\varepsilon}$ coincides with the subspace described in the statement of the theorem.

The construction of the subspace of incoming scattering states $\mathcal{H}^{-}$and of the corresponding projections $Q^{-}$proceeds analogously as above, by taking the limit $t \rightarrow-\infty$. Assuming that $\mathbf{1}_{(-\infty, E]}(H) \mathcal{H}^{+}=\mathbf{1}_{(-\infty, E]}(H) \mathcal{H}^{-}$for some $E \leq \Sigma$, (which holds under conditions specified in [23] as a consequence of asymptotic completeness) transition probabilities for inclusive collision processes below this energy can be defined using formula (1.9).

\section{Conclusion and outlook}

In this work we made first steps towards a construction of inclusive collision crosssections in the massless Nelson model, following ideas developed in algebraic quantum field theory $[8]$. We identified suitable asymptotic observables which play the role of particle detectors. Their coincidence arrangements can be used for preparation of incoming and outgoing configurations of hard particles accompanied by some unspecified configurations of soft photons.

We tested the proposed construction in the absence of the infrared problem, to show that it is consistent with predictions of standard scattering theory. As 
for the infrared-singular case, the first question is the existence of the particle detectors $C_{g}^{+}(\chi)$ and $C^{+}(f)$. In the case of the photon detector, a positive answer can be inferred from the existing literature: Using methods developed in [14, the convergence of $t \rightarrow \mathrm{e}^{i H t} \mathrm{~d} \Gamma(\chi(y / t)) \mathrm{e}^{-i H t}$ is established in [23. for the Nelson model with an infrared cut-off. The convergence of $t \rightarrow \mathrm{e}^{i H t} \mathrm{~d} \Gamma(g \chi(y / t) g) \mathrm{e}^{-i H t}$, where $g$ vanishes in a neighbourhood of zero, can be established analogously in the absence of the infrared cut-off. Thus, by a minor modification of the proof of Theorem 26 of [23], one obtains:

Theorem 5.1. Let $\lambda, \Sigma$ be s.t. $\left\||\nabla \Omega(p)| \mathbf{1}_{(-\infty, \Sigma]}(H)\right\| \leq \beta$ for some $\beta<1$. Let $\chi \in C_{0}^{\infty}\left(\mathbb{R}^{3}\right)_{\mathbb{R}}$ be s.t. $\operatorname{supp} \chi \subset\left\{v \in \mathbb{R}^{3}|| v \mid>\beta\right\}$ and suppose that $g \in C_{0}^{\infty}(\mathbb{R})_{\mathbb{R}}$ vanishes in a neighbourhood of zero. Then the limit

$$
C_{g}^{+}(\chi) \Psi:=\mathrm{s}-\lim _{t \rightarrow \infty} \mathrm{e}^{i H t} \mathrm{~d} \Gamma(g \chi(y / t) g) \mathrm{e}^{-i H t} \Psi
$$

exists for any $\Psi \in \operatorname{Ran}_{(-\infty, \Sigma)}(H)$.

The problem of existence of $C^{+}(f)=\mathrm{s}-\lim _{t \rightarrow \infty} \mathrm{e}^{i H t} f(x / t) \mathrm{e}^{-i H t}$ in the presence of the infrared problem appears to be more difficult. To our knowledge, it is only resolved on the subspace of infraparticle scattering states, constructed in [32, 33]. We recall that an analogous asymptotic observable (asymptotic velocity) plays a central role in quantum mechanical scattering theory, where it exists under very general conditions [13. There the main ingredient of the proof of convergence is the equality of the average velocity $x / t$ and the instantaneous velocity $p / M$ of the particle at asymptotic times (Graf's propagation estimate [25]). This route seems difficult in the setting of non-relativistic QED due to the phenomenon of the electron mass renormalization. We hope, however, that recently developed powerful time dependent [16] and spectral [9] methods will shed some light on the question of existence of the asymptotic velocity of the electron in models of non-relativistic QED. In view of the framework proposed in the present paper, an answer to this question will allow for a meaningful definition of inclusive collision cross-sections in these models. It may also clarify the problem of convergence of the particle detector approximants (1.1) and thus contribute to the understanding of the infrared problem in relativistic (algebraic) quantum field theory.

Acknowledgements: I would like to thank D. Buchholz, J. Dereziński, A. Pizzo, W. De Roeck and H. Spohn for interesting discussions on scattering theory. The hospitality extended to me at final stages of this work by the University of Heidelberg and the University of California, Davis is gratefully acknowledged. 


\section{A Proof of Proposition 3.1}

In this Appendix we give a proof of Proposition 3.1. More technical part of this discussion is postponed to subsequent lemmas.

Proof of Proposition 3.1: Making use of Lemma 2.4, we obtain

$$
\begin{aligned}
\mathrm{e}^{i H t} f(x / t) \mathrm{e}^{-i H t} a_{+}^{*}\left(h_{1}\right) & \ldots a_{+}^{*}\left(h_{n}\right) \Psi \\
& =\mathrm{e}^{i H t} f(x / t) \mathrm{e}^{-i H t} g^{\prime}(H) a_{+}^{*}\left(h_{1}\right) \ldots a_{+}^{*}\left(h_{n}\right) \Psi
\end{aligned}
$$

for some $g^{\prime} \in C_{0}^{\infty}(\mathbb{R})$. There holds

$$
\begin{array}{r}
\mathrm{e}^{i H t} f(x / t) g(H) a^{*}\left(h_{1, t}\right) \ldots a^{*}\left(h_{n, t}\right) \Psi_{t}=\mathrm{e}^{i H t}\left[f(x / t), g^{\prime}(H)\right] a^{*}\left(h_{1, t}\right) \ldots a^{*}\left(h_{n, t}\right) \Psi_{t} \\
+\mathrm{e}^{i H t} g^{\prime}(H) a^{*}\left(h_{1, t}\right) \ldots a^{*}\left(h_{n, t}\right) f(x / t) \Psi_{t}, \quad(\mathrm{~A} .2)
\end{array}
$$

where $\Psi_{t}=\mathrm{e}^{-i H t} \Psi$. The first term on the r.h.s. tends to zero as $t \rightarrow \infty$ by Lemmas A.2 and 2.2. To treat the second term, we choose a real-valued function $\tilde{E} \in C_{0}^{\infty}\left(\mathbb{R}^{3}\right)$ which coincides with $\xi \rightarrow E(\xi)$ for $E(\xi) \leq \Sigma$. We write

$$
\begin{aligned}
& \mathrm{e}^{i H t} g^{\prime}(H) a^{*}\left(h_{1, t}\right) \ldots a^{*}\left(h_{n, t}\right) f(x / t) \Psi_{t} \\
= & \mathrm{e}^{i H t} g^{\prime}(H) a^{*}\left(h_{1, t}\right) \ldots a^{*}\left(h_{n, t}\right) \mathrm{e}^{-i \tilde{E}(P) t}\left(\mathrm{e}^{i \tilde{E}(P) t} f(x / t) \mathrm{e}^{-i \tilde{E}(P) t} \Psi-f(\nabla \tilde{E}(P)) \Psi\right) \\
+ & \mathrm{e}^{i H t} g^{\prime}(H) a^{*}\left(h_{1, t}\right) \ldots a^{*}\left(h_{n, t}\right) \mathrm{e}^{-i H t} f(\nabla \tilde{E}(P)) \Psi .
\end{aligned}
$$

Since $\left\|g^{\prime}(H) a^{*}\left(h_{1, t}\right) \ldots a^{*}\left(h_{n, t}\right)\right\|$ is bounded uniformly in time, (see Lemma 2.2), the first term on the r.h.s. above converges to zero, by Lemma A.3 with the above choice of the function $\tilde{E}$. The second term converges to the r.h.s. of (3.3) by Lemma 2.4. This concludes the proof.

In the proof of the auxiliary Lemma A.2, stated below, there enter two ingredients. The first is a simple fact from the pseudodifferential calculus, whose proof can be found in 22$]$.

Lemma A.1. Let $f \in S\left(\mathbb{R}^{3}\right), h \in C^{n}\left(\mathbb{R}^{3}\right)$ and $\sup _{|\alpha|=2}\left\|\partial^{\alpha} h\right\|_{\infty}<\infty$, where $\partial^{\alpha}=\partial_{1}^{\alpha_{1}} \partial_{2}^{\alpha_{2}} \partial_{3}^{\alpha_{3}}$ and $|\alpha|=\alpha_{1}+\alpha_{2}+\alpha_{3}$. Then

$$
\begin{aligned}
i[h(k), f(\varepsilon x)] & =i \varepsilon \nabla h(k) \cdot \nabla f(\varepsilon x)+R_{1, t}(\varepsilon) \\
& =i \varepsilon \nabla f(\varepsilon x) \cdot \nabla h(k)+R_{2, t}(\varepsilon),
\end{aligned}
$$

where

$$
\left\|R_{j, t}(\varepsilon)\right\| \leq C \varepsilon^{2} \sup _{|\alpha|=2}\left\|\partial^{\alpha} h\right\|_{\infty} \int_{\mathbb{R}^{3}} d u|u|^{2}|\hat{f}(u)|
$$

for some constant $C$ independent of $\varepsilon$.

The second ingredient is the Helffer-Sjöstrand functional calculus, which we summarize following $\left[22\right.$ : Let $f \in C_{0}^{\infty}(\mathbb{R}, \mathbb{C})$ and $A$ be a self-adjoint operator. Then $f(A)$ can be represented as follows

$$
f(A)=-\frac{1}{\pi} \int d u d v \partial_{\bar{z}} \tilde{f}(z)(z-A)^{-1}, \quad z=u+i v .
$$


This holds for any function $\tilde{f} \in C_{0}^{\infty}\left(\mathbb{R}^{2}, \mathbb{C}\right)$ s.t. $\left|\partial_{\bar{z}} \tilde{f}(z)\right| \leq C|v|, \tilde{f}(z)=f(z)$ and $\partial_{\bar{z}} \tilde{f}(z)=\frac{1}{2}\left(\partial_{u} f+i \partial_{w} f\right)(z)=0$ for all $z \in \mathbb{R}$. Such $\tilde{f}$ is called an almost-analytic extension of $f$. For any $n \in \mathbb{N}$ there exist extensions for which $\left|\partial_{\bar{z}} \tilde{f}(z)\right| \leq C|v|^{n}$.

Lemma A.2. For any $f \in C_{0}^{\infty}\left(\mathbb{R}^{3}\right)$ and $h \in C_{0}^{\infty}(\mathbb{R})$ there holds

$$
\|[h(H), f(x / t)]\| \leq C / t
$$

for some constant $C \geq 0$ independent of $t$.

Proof. We choose an almost-analytic extension of $h$ s.t $\left|\partial_{\bar{z}} \tilde{h}(u, v)\right| \leq C|v|^{2}$. Then, for $z=u+i v$

$$
\begin{aligned}
& {[h(H), f(x / t)]} \\
& =-\frac{1}{\pi} \int d u d v \partial_{\bar{z}} \tilde{h}(u, v)\left[(z-H)^{-1}, f(x / t)\right] \\
& =-\frac{1}{\pi} \int d u d v \partial_{\bar{z}} \tilde{h}(u, v)(z-H)^{-1}[\Omega(p), f(x / t)](z-H)^{-1} .
\end{aligned}
$$

Now by Lemma A.1, $[\Omega(p), f(x / t)]=\frac{1}{t}(\nabla f)(x / t) \nabla \Omega(p)+R_{2}(t)$, where $\left\|R_{2}(t)\right\| \leq$ $c^{\prime} / t^{2}$. We note the following estimate

$$
\left\|\partial_{i} \Omega(p)(z-H)^{-1}\right\| \leq\left\|\partial_{i} \Omega(p)(c+H)^{-\frac{1}{2}}\right\|\left\|(c+H)^{\frac{1}{2}}(z-H)^{-1}\right\|,
$$

where $c \geq 0$ is chosen s.t. $\sigma(H)+c \subset(0, \infty)$. The first factor on the r.h.s. of (A.9) above can be estimated as follows:

$$
\begin{aligned}
\left\|\partial_{i} \Omega(p)(c+H)^{-\frac{1}{2}}\right\|^{2} & =\left\|(c+H)^{-\frac{1}{2}}\left|\partial_{i} \Omega(p)\right|^{2}(c+H)^{-\frac{1}{2}}\right\| \\
& \leq(2 / M)\left\|(c+H)^{-\frac{1}{2}}(\Omega(p))(c+H)^{-\frac{1}{2}}\right\| \\
& \leq(2 / M)\left\|\left(H+c_{1}\right)(c+H)^{-1}\right\|,
\end{aligned}
$$

where the bound $\Omega(p) \leq H+c_{1}$ for some constant $c_{1}$ follows e.g. from Lemma 8 of [23]. The second factor on the r.h.s. of (A.9) gives

$$
\begin{aligned}
\left\|(c+H)^{\frac{1}{2}}(z-H)^{-1}\right\|^{2} & =\left\|(\bar{z}+H)^{-1}(c+H)(z+H)^{-1}\right\| \\
& =\sup _{w \geq 0} \frac{w}{(u+w-c)^{2}+v^{2}} \\
& =\sup _{w \geq R} \frac{w}{(u+w-c)^{2}+v^{2}} \\
& +\sup _{0 \leq w \leq R} \frac{w}{(u+w-c)^{2}+v^{2}},
\end{aligned}
$$

where $R \geq \sup \left\{|u| \mid \partial_{\bar{z}} \tilde{g}(u, \cdot) \equiv 0\right\}+c+1$. The second term on the r.h.s. of (A.11) can be estimated by $R / v^{2}$ and the first one by $\sup _{w \geq R} w /(w-R+1)^{2} \leq C$. Hence,

$$
\left\|\partial_{i} \Omega(p)(z-H)^{-1}\right\| \leq C /|v| .
$$

Making use of the above facts and of the obvious inequality $\left\|(z-H)^{-1}\right\| \leq C /|v|$, we complete the proof.

Let us now proceed to the second auxiliary lemma which we used in the proof of Proposition 3.1. 
Lemma A.3. Let $f \in C_{0}^{\infty}\left(\mathbb{R}^{3}\right)$ and $\tilde{E} \in C_{0}^{\infty}\left(\mathbb{R}^{3}\right)$ be a real-valued function. Then

$$
\text { S- } \lim _{t \rightarrow \infty} \mathrm{e}^{i \tilde{E}(P) t} f(x / t) \mathrm{e}^{-i \tilde{E}(P) t}=f(\nabla \tilde{E}(P)) .
$$

Proof. Let $\Psi \in \mathcal{H}$. By the functional calculus for a family of commuting selfadjoint operators, we obtain

$$
\mathrm{e}^{i \tilde{E}(P) t} f(x / t) \mathrm{e}^{-i \tilde{E}(P) t} \Psi=f(x / t+\nabla \tilde{E}(P)) \Psi .
$$

We note that, by Theorem VIII.25 of [36], $x^{i} / t+\partial_{i} \tilde{E}(P)$ converges to $\partial_{i} \tilde{E}(P)$ in the strong resolvent sense. In fact, the domain of $x^{i}$, denoted $D\left(x^{i}\right)$, is a common core for all the approximants and the limit, and for any $\Phi \in D\left(x^{i}\right)$

$$
\text { S- } \lim _{t \rightarrow \infty}\left(x^{i} / t+\partial_{i} \tilde{E}(P)\right) \Phi=\partial_{i} \tilde{E}(P) \Phi .
$$

Thus we obtain A.13) e.g. by approximating $f$ in the supremum norm by functions of the form $f_{\varepsilon}(x)=\sum_{j} f_{\varepsilon, j, 1}\left(x^{1}\right) f_{\varepsilon, j, 2}\left(x^{2}\right) f_{\varepsilon, j, 3}\left(x^{3}\right)$, where $f_{\varepsilon, j, k} \in S(\mathbb{R})$ and the sum is finite.

\section{B Proof of Proposition 3.2}

In this Appendix we prove Proposition 3.2 which establishes the existence of the photon detectors on scattering states of bounded energy. As in Appendix A, the more technical part of this discussion is given in subsequent lemmas.

Proof of Proposition 3.2: By Lemma 2.4, we obtain

$$
\begin{aligned}
\mathrm{e}^{i H t} \mathrm{~d} \Gamma & (g \chi(y / t) g) \mathrm{e}^{-i H t} a_{+}^{*}\left(h_{1}\right) \ldots a_{+}^{*}\left(h_{n}\right) \Psi \\
& =\mathrm{e}^{i H t} \mathrm{~d} \Gamma(g \chi(y / t) g) \mathrm{e}^{-i H t} g^{\prime}(H) a_{+}^{*}\left(h_{1}\right) \ldots a_{+}^{*}\left(h_{n}\right) \Psi \\
& =\mathrm{e}^{i H t}\left[\mathrm{~d} \Gamma(g \chi(y / t) g), g^{\prime}(H)\right] \mathrm{e}^{-i H t} a_{+}^{*}\left(h_{1}\right) \ldots a_{+}^{*}\left(h_{n}\right) \Psi \\
& +\mathrm{e}^{i H t} g^{\prime}(H) \mathrm{d} \Gamma(g \chi(y / t) g) \mathrm{e}^{-i H t} a_{+}^{*}\left(h_{1}\right) \ldots a_{+}^{*}\left(h_{n}\right) \Psi
\end{aligned}
$$

for some $g^{\prime} \in C_{0}^{\infty}(\mathbb{R})$. It follows from Lemma B.1 and Proposition 3.1 that the term with the commutator tends to zero. As for the last term on the r.h.s. of (B.1), we note that

$$
\sup _{t}\left\|g^{\prime}(H) \mathrm{d} \Gamma(g \chi(y / t) g)\right\|<\infty .
$$

This is a consequence of Lemmas C.1 and 2.2 (a). Thus we can write

$$
\begin{aligned}
& g^{\prime}(H) \mathrm{e}^{i H t} \mathrm{~d} \Gamma(g \chi(y / t) g) \mathrm{e}^{-i H t} a_{+}^{*}\left(h_{1}\right) \ldots a_{+}^{*}\left(h_{n}\right) \Psi \\
&=g^{\prime}(H) \mathrm{e}^{i H t} \mathrm{~d} \Gamma(g \chi(y / t) g) a^{*}\left(h_{1, t}\right) \ldots a^{*}\left(h_{n, t}\right) \Psi_{t}+o(1) \\
&=g^{\prime}(H) \mathrm{e}^{i H t} \sum_{j=1}^{n} a^{*}\left(h_{1, t}\right) \ldots a^{*}\left(g \chi(y / t) g h_{j, t}\right) \ldots a^{*}\left(h_{n, t}\right) \Psi_{t} \\
&+g^{\prime}(H) \mathrm{e}^{i H t} a^{*}\left(h_{1, t}\right) \ldots a^{*}\left(h_{n, t}\right) \mathrm{d} \Gamma(g \chi(y / t) g) \Psi_{t}+o(1)
\end{aligned}
$$


where $o(1)$ denotes a rest term which tends to zero in norm as $t \rightarrow \infty$ and we used that $\left[\mathrm{d} \Gamma(g \chi(y / t) g), a^{*}\left(h_{j, t}\right)\right]=a^{*}\left(g \chi(y / t) g h_{j, t}\right)$. Let us first study one of the terms in the sum above. We will show that $a^{*}\left(g \chi(y / t) g h_{j, t}\right)$ can be replaced with $a^{*}\left(\left\{g^{2} \chi(\nabla \omega) h_{j}\right\}_{t}\right)$ at a cost of an error term of order $o(1)$. We set

$$
\tilde{h}_{j, t}:=\mathrm{e}^{-i \omega t} g\left(\mathrm{e}^{i \widetilde{\omega} t} \chi(y / t) \mathrm{e}^{-i \widetilde{\omega} t}-\chi(\nabla \widetilde{\omega})\right) g h_{j},
$$

where $\widetilde{\omega}$ is a smooth, compactly supported function which coincides with $\omega$ on the support of $g$. We obtain

$$
\begin{aligned}
& \left\|g^{\prime}(H) \mathrm{e}^{i H t} a^{*}\left(h_{1, t}\right) \ldots a^{*}\left(\tilde{h}_{j}\right) \ldots a^{*}\left(h_{n, t}\right) \Psi_{t}\right\| \\
= & \left\|g^{\prime}(H) \mathrm{e}^{i H t} a^{*}\left(h_{1, t}\right) \ldots \check{j} \ldots a^{*}\left(h_{n, t}\right) a^{*}\left(\tilde{h}_{j, t}\right) \Psi_{t}\right\| \\
\leq & \left\|g^{\prime}(H) \mathrm{e}^{i H t} a^{*}\left(h_{1, t}\right) \ldots \check{j} \ldots a^{*}\left(h_{n, t}\right)\right\|\left\|a^{*}\left(\tilde{h}_{j, t}\right) g^{\prime}(H)\right\|\|\Psi\|,
\end{aligned}
$$

where $\check{j}$ denotes the omission of the $j$-th creation operator. By Lemma 2.2 the first factor on the r.h.s. above is bounded uniformly in time, whereas the second factor satisfies

$$
\left\|a^{*}\left(\tilde{h}_{j, t}\right) g^{\prime}(H)\right\| \leq C\left\|\left(1+\omega^{-1}\right)^{\frac{1}{2}} \tilde{h}_{j, t}\right\|,
$$

for some constant $C \geq 0$. Denoting $\tilde{g}(q)=\left(1+\omega(q)^{-1}\right)^{\frac{1}{2}} g(q)$, we obtain

$$
\left\|\left(1+\omega^{-1}\right)^{\frac{1}{2}} \tilde{h}_{j, t}\right\| \leq\|\tilde{g}\|_{\infty}\left\|\left(\mathrm{e}^{i \widetilde{\omega} t} \chi(y / t) \mathrm{e}^{-i \widetilde{\omega} t}-\chi(\nabla \widetilde{\omega})\right) g h_{j}\right\| .
$$

Proceeding as in the proof of Lemma A.3, we obtain

$$
\text { S- } \lim _{t \rightarrow \infty} \mathrm{e}^{i \widetilde{\omega} t} \chi(y / t) \mathrm{e}^{-i \widetilde{\omega} t}=\chi(\nabla \widetilde{\omega}) .
$$

Hence, we have shown that

$$
\begin{aligned}
& \sum_{j=1}^{n} g^{\prime}(H) \mathrm{e}^{i H t} a^{*}\left(h_{1, t}\right) \ldots a^{*}\left(g \chi(y / t) g h_{j, t}\right) \ldots a^{*}\left(h_{n, t}\right) \Psi_{t} \\
= & \sum_{j=1}^{n} g^{\prime}(H) \mathrm{e}^{i H t} a^{*}\left(h_{1, t}\right) \ldots a^{*}\left(\left\{g^{2} \chi(\nabla \widetilde{\omega}) h_{j}\right\}_{t}\right) \ldots a^{*}\left(h_{n, t}\right) \Psi_{t}+o(1) .
\end{aligned}
$$

Making use of Lemma 2.4 and exploiting the fact that $|\nabla \widetilde{\omega}(q)|=1$ for $q \in \operatorname{supp} g$, we obtain in the limit $t \rightarrow \infty$ the r.h.s. of (3.4).

It still has to be shown that the remaining terms on the r.h.s. of $(\underline{B .3})$ tend to zero. To this end, we write

$$
\begin{aligned}
& \left\|g^{\prime}(H) \mathrm{e}^{i H t} a^{*}\left(h_{1, t}\right) \ldots a^{*}\left(h_{n, t}\right) \mathrm{d} \Gamma(g \chi(y / t) g) \Psi_{t}\right\| \\
& \leq\left\|g^{\prime}(H) \mathrm{e}^{i H t} a^{*}\left(h_{1, t}\right) \ldots a^{*}\left(h_{n, t}\right)\right\|\left\|\mathrm{d} \Gamma(g \chi(y / t) g) \Psi_{t}\right\| .
\end{aligned}
$$

Here the first factor on the r.h.s. is uniformly bounded by Lemma 2.2. The second factor vanishes for $t \rightarrow \infty$ by Lemma B.2. $\square$

In the above proof we used the following two lemmas: 
Lemma B.1. Let $f \in C_{0}^{\infty}\left(\mathbb{R}^{3}\right)$ be s.t. $\operatorname{supp} f \subset\left\{u \in \mathbb{R}^{3}|| u \mid \leq \beta\right\}$. Choose $\chi \in C_{0}^{\infty}\left(\mathbb{R}^{3}\right)$ s.t. $\operatorname{supp} \chi \subset\left\{v \in \mathbb{R}^{3}|| v \mid \geq \beta+\varepsilon\right\}$ for some $\beta, \varepsilon>0$. Then

$$
\begin{aligned}
& \left\|\left[\mathrm{d} \Gamma(g \chi(y / t) g), g^{\prime}(H)\right]\right\| \leq C, \\
& \left\|\left[\mathrm{~d} \Gamma(g \chi(y / t) g), g^{\prime}(H)\right] f(x / t)\right\| \leq C / t,
\end{aligned}
$$

for $c$ independent of $t, g^{\prime} \in C_{0}^{\infty}(\mathbb{R})$ and $g \in C_{0}^{\infty}\left(\mathbb{R}^{3}\right)_{\mathbb{R}}$ s.t. $g$ vanishes in a neighbourhood of zero.

Proof. We apply the Helffer-Sjöstrand functional calculus (see Appendix A). Choosing an almost-analytic extension $\tilde{g}^{\prime}$ of $g^{\prime}$ s.t. $\left|\partial_{\bar{z}} \tilde{g}^{\prime}(u, v)\right| \leq C|v|^{3}$, we obtain

$$
\begin{aligned}
i\left[\mathrm{~d} \Gamma(g \chi(y / t) g), g^{\prime}(H)\right] & \\
= & -\frac{1}{\pi} \int d u d v \partial_{\bar{z}} \tilde{g}^{\prime}(u, v)(z-H)^{-1} \mathrm{~d} \Gamma(g i[\widetilde{\omega}, \chi(y / t)] g)(z-H)^{-1} \\
& +\frac{1}{\pi} \int d u d v \partial_{\bar{z}} \tilde{g}^{\prime}(u, v)(z-H)^{-1} \phi\left(i g \chi(y / t) g G_{x}\right)(z-H)^{-1},
\end{aligned}
$$

where $z=u+i v, \widetilde{\omega}$ is a smooth, compactly supported function which coincides with $\omega$ on the support of $g$. To obtain (B.13), we used

$$
\begin{aligned}
& i\left[\mathrm{~d} \Gamma(g \chi(y / t) g), \phi\left(G_{x}\right)\right]=\phi\left(i g \chi(y / t) g G_{x}\right), \\
& i[\mathrm{~d} \Gamma(g \chi(y / t) g), \mathrm{d} \Gamma(\omega)]=\mathrm{d} \Gamma(i[g \chi(y / t) g, \omega]) .
\end{aligned}
$$

Next, we denote by $C_{1, t}, C_{2, t}$ the first and the second term on the r.h.s. of (B.13), respectively. First, we show that

$$
\left\|C_{1, t}\right\| \leq C / t .
$$

To this end, we note that for $c \geq 0$ s.t. $\sigma(H)+c \subset(0, \infty)$

$$
\begin{aligned}
(c+H)^{-\frac{1}{2}} \mathrm{~d} \Gamma(g i[\widetilde{\omega}, \chi(y / t)] g)(c+H)^{-\frac{1}{2}} & \\
\leq & \frac{C}{t}(c+H)^{-\frac{1}{2}} \mathrm{~d} \Gamma\left(g^{2}\right)(c+H)^{-\frac{1}{2}}+O\left(t^{-2}\right) \\
& \leq \frac{C^{\prime}}{t}\left(c_{1} H+c_{2}\right)(c+H)^{-1}+O\left(t^{-2}\right),
\end{aligned}
$$

where we made use of the pseudodifferential calculus (cf. Lemma A.1) and of the fact that $\mathrm{d} \Gamma\left(b_{1}\right) \leq \mathrm{d} \Gamma\left(b_{2}\right)$ for any self-adjoint operators $b_{1}, b_{2}$ s.t. $b_{1} \leq b_{2}$. The term $O\left(t^{-2}\right)$ denotes a family of bounded operators s.t. $\left\|O\left(t^{-2}\right)\right\| \leq C t^{-2}$ for some constant $C$. Noting, as in the proof of Lemma A.2, that $\left\|(c+H)^{\frac{1}{2}}(z-H)^{-1}\right\| \leq$ $C /|v|$, we obtain (B.16).

As for $C_{2, t}$, we first show that it is bounded uniformly in $t$. To this end, we note that, by Lemma 2.2 (b),

$$
\begin{aligned}
\|\left(1+H_{\mathrm{ph}}\right)^{-\frac{1}{2}} \phi & \left(i g \chi(y / t) g G_{x}\right)\left(1+H_{\mathrm{ph}}\right)^{-\frac{1}{2}} \| \\
& \leq C \sup _{x}\left\|\left(1+\omega^{-1}\right)^{\frac{1}{2}} g \chi(y / t) g G_{x}\right\| \\
& \leq C\left\|\left(1+\omega^{-1}\right)^{\frac{1}{2}} g\right\|\|\chi\|_{\infty}\|g\|_{\infty}\|G\| .
\end{aligned}
$$


Exploiting the fact that $\left\|\left(1+H_{\mathrm{ph}}\right)^{\frac{1}{2}}(z-H)^{-1}\right\| \leq c /|v|$, we obtain that $\left\|C_{2, t}\right\|<\infty$ uniformly in $t$. This concludes the proof of (B.11). To verify (B.12), we still have to check that $\left\|C_{2, t} f(x / t)\right\| \leq C / t$ as $t \rightarrow \infty$. Setting $h_{x}:=i g \chi(y / t) g G_{x}$, we obtain

$$
\begin{aligned}
(z-H)^{-1} \phi\left(h_{x}\right) & (z-H)^{-1} f(x / t) \\
& =(z-H)^{-1} \phi\left(h_{x}\right)(z-H)^{-1}[\Omega(p), f(x / t)](z-H)^{-1} \\
& +(z-H)^{-1} \phi\left(h_{x}\right) f(x / t)(z-H)^{-1} .
\end{aligned}
$$

By Lemma A.1, $i[\Omega(p), f(x / t)]=\frac{1}{t} \nabla f(x / t) \nabla \Omega(p)+R_{t}$, where $\left\|R_{t}\right\| \leq C / t^{2}$. We note that $\left\||\nabla \Omega(p)|(c+H)^{-\frac{1}{2}}\right\|<\infty$ and $\left\|(c+H)^{\frac{1}{2}}(z-H)^{-1}\right\| \leq C /|v|$. From the latter inequality and from (B.18) we obtain $\sup _{x}\left\|(z-H)^{-1} \phi\left(h_{x}\right)(z-H)^{-1}\right\| \leq$ $C /|v|^{2}$. Hence the contribution to $C_{2, t}$, corresponding to the first term on the r.h.s. of (B.19), is bounded by $C / t$. To estimate the second term on the r.h.s. of (B.19), we note that

$$
\begin{aligned}
\|\left(1+H_{\mathrm{ph}}\right)^{-\frac{1}{2}} \phi\left(h_{x}\right) & f(x / t)\left(1+H_{\mathrm{ph}}\right)^{-\frac{1}{2}} \| \\
& \leq C\left\|\left(1+\omega^{-1}\right)^{\frac{1}{2}} g\right\|_{\infty} \sup _{|x| / t \leq \beta}\left\|\chi(y / t) g G_{x}\right\| .
\end{aligned}
$$

To show that this expression tends to zero, we recall that $G_{x}(q)=\kappa(q) \mathrm{e}^{-i q x}$, where $\kappa(q)=\lambda \frac{\hat{\rho}(q)}{\sqrt{2 \omega(q)}}, \hat{\rho} \in C_{0}^{\infty}\left(\mathbb{R}^{3}\right)$ and obtain, similarly as in Lemma 9 of [23],

$$
\begin{aligned}
\sup _{|x| / t \leq \beta}\left\|\chi(y / t) g G_{x}\right\|^{2} & \leq \sup _{|x| / t \leq \beta} \int_{|y| / t \geq \beta+\varepsilon} d y|\widehat{g \kappa}(y-x)|^{2} \\
& \leq\left(\frac{1}{\varepsilon t}\right)^{n} \int d y|\widehat{g \kappa}(y)|^{2}|y|^{n} .
\end{aligned}
$$

The integral on the r.h.s. is finite for any $n \geq 0$, since $g \kappa \in C_{0}^{\infty}\left(\mathbb{R}^{3}\right)$. Using the estimate $\left\|\left(1+H_{\mathrm{ph}}\right)^{\frac{1}{2}}(z-H)^{-1}\right\|^{2} \leq c /|v|^{2}$, we conclude that $\left\|C_{2, t} f(x / t)\right\| \leq C / t$ as $t \rightarrow \infty$.

Lemma B.2. Let $\varepsilon>0$ be s.t. $|\nabla E(\xi)|<1-\varepsilon$ for $E(\xi) \leq \Sigma$. (Cf. Lemma 2.1). Then, for any $\chi \in C_{0}^{\infty}\left(\mathbb{R}^{3}\right)$ s.t. $\operatorname{supp} \chi \subset\left\{v \in \mathbb{R}^{3}|||v|-1 \mid \leq \varepsilon / 4\right\}$ and $\Psi \in \mathcal{H}_{\text {des }}$,

$$
\lim _{t \rightarrow \infty}\left\|\mathrm{d} \Gamma(g \chi(y / t) g) \Psi_{t}\right\|=0,
$$

where $\Psi_{t}=\mathrm{e}^{-i H t} \Psi$ and $g \in C_{0}^{\infty}\left(\mathbb{R}^{3}\right)_{\mathbb{R}}$ vanishes in a neighbourhood of zero.

Proof. We choose a real-valued function $\tilde{E} \in C_{0}^{\infty}\left(\mathbb{R}^{3}\right)$ which coincides with $\xi \rightarrow E(\xi)$ for $E(\xi) \leq \Sigma$ and satisfies $|\nabla \tilde{E}(\xi)|<1-\varepsilon$ for all $\xi \in \mathbb{R}^{3}$. As $\Psi$ has bounded energy, we can choose $g^{\prime} \in C_{0}^{\infty}(\mathbb{R})$ s.t. $\Psi=g^{\prime}(H) \Psi$. Since, by Lemma B.1 and Proposition 3.1, $\left[\mathrm{d} \Gamma(g \chi(y / t) g), g^{\prime}(H)\right] \Psi_{t}$ tends strongly to zero as $t \rightarrow \infty$, we obtain

$$
\begin{array}{r}
\left\|\mathrm{d} \Gamma(g \chi(y / t) g) \Psi_{t}\right\|=\left\|g^{\prime}(H) \mathrm{e}^{i \tilde{E}(P) t} \mathrm{~d} \Gamma(g \chi(y / t) g) \mathrm{e}^{-i \tilde{E}(P) t} \Psi\right\|+o(1) \\
\leq c\left\|\mathrm{e}^{i \tilde{E}(P) t}\left(1+H_{\mathrm{ph}}\right)^{-\frac{1}{2}} \mathrm{~d} \Gamma(g \chi(y / t) g)\left(1+H_{\mathrm{ph}}\right)^{-\frac{1}{2}} \mathrm{e}^{-i \tilde{E}(P) t} \Psi_{\mathrm{ph}}\right\|+o(1),
\end{array}
$$


where we set $\Psi_{\mathrm{ph}}=\left(1+H_{\mathrm{ph}}\right)^{\frac{1}{2}} \Psi$ and the constant $c$ is independent of $t$. We decompose $\Psi_{\mathrm{ph}}=\sum_{m} \Psi_{\mathrm{ph}, m}$ into components with fixed photon number and write

$$
\begin{gathered}
\left\|\mathrm{e}^{i \tilde{E}(P) t}\left(1+H_{\mathrm{ph}}\right)^{-\frac{1}{2}} \mathrm{~d} \Gamma(g \chi(y / t) g)\left(1+H_{\mathrm{ph}}\right)^{-\frac{1}{2}} \mathrm{e}^{-i \tilde{E}(P) t} \Psi_{\mathrm{ph}}\right\|^{2} \\
=\sum_{m}\left\|\left(1+H_{\mathrm{ph}}\right)^{-\frac{1}{2}} \sum_{j=1}^{m} g\left(k_{j}\right) \chi\left(y_{j} / t+\nabla \tilde{E}(P)\right) g\left(k_{j}\right)\left(1+H_{\mathrm{ph}}\right)^{-\frac{1}{2}} \Psi_{\mathrm{ph}, m}\right\|^{2},
\end{gathered}
$$

where we identified $\Psi_{\mathrm{ph}, m}$ with (classes of) square-integrable functions on $\mathbb{R} \times \mathbb{R}^{3 m}$ and made use of the fact that $\mathrm{e}^{i \tilde{E}(P) t} y_{j}^{i} \mathrm{e}^{-i \tilde{E}(P) t}=y_{j}^{i}+\partial_{i} \tilde{E}(P) t$ on a suitable dense domain in $L^{2}\left(\mathbb{R}^{3}\right) \otimes L^{2}\left(\mathbb{R}^{3}\right)^{\otimes m}$. In order to enter with the limit $t \rightarrow \infty$ under the sum, we show that the above sequence can be estimated by a uniformly convergent sequence independent of $t$ and make use of the dominated convergence theorem. To this end, we estimate in the $m$-particle subspace

$$
\begin{aligned}
& \left(1+H_{\mathrm{ph}}\right)^{-\frac{1}{2}} \sum_{j=1}^{m} g\left(k_{j}\right) \chi\left(y_{j} / t+\nabla \tilde{E}(P)\right) g\left(k_{j}\right)\left(1+H_{\mathrm{ph}}\right)^{-\frac{1}{2}} \\
& \leq\|\chi(y / t+\nabla \tilde{E}(P))\|\left\|g(k)^{2}|k|^{-1}\right\| \sum_{j=1}^{m} \omega\left(k_{j}\right)\left(1+H_{\mathrm{ph}}\right)^{-1} \\
& \leq C\left(1+H_{\mathrm{ph}}\right)^{-1} H_{\mathrm{ph}} \leq C^{\prime} .
\end{aligned}
$$

Clearly, $C^{\prime}$ can be chosen uniformly in $t$ and $m$. Thus we can enter with the limit under the sum in (B.24). The expression tends to zero because $y_{j} / t+\partial_{i} \tilde{E}(P)$ tends to $\partial_{i} E(P)$ in the strong resolvent sense and $\nabla \tilde{E}(P)$ has its spectrum outside of the support of $\chi$. (Cf. the proof of Lemma A.3).

\section{Proof of Proposition 3.3}

Proof of Proposition 3.3: The invariance of $C^{+}(f)$ follows from the the estimate

$$
\begin{aligned}
\|f(x /(t-s))-f(x / t)\| & =\left\|\int_{0}^{s} \frac{d}{d s^{\prime}} f\left(x /\left(t-s^{\prime}\right)\right) d s^{\prime}\right\| \\
& \leq \frac{s}{(t-s)} \sup _{v \in \mathbb{R}^{3}}|\nabla f(v) \cdot v|,
\end{aligned}
$$

where the r.h.s. tends to zero as $t \rightarrow \infty$.

The invariance of $C_{g}^{+}(\chi)$ can be proven by an analogous argument (cf. the proof of Theorem 26 of [23]): We note that

$$
\begin{aligned}
\left\|\mathrm{d} \Gamma(g(\chi(y /(t-s))-\chi(y / t)) g)(c+H)^{-1}\right\| & \leq c^{\prime}\left\|\int_{0}^{s} d s^{\prime} \frac{d}{d s^{\prime}} \chi\left(y /\left(t-s^{\prime}\right)\right)\right\| \\
& \leq c^{\prime} \frac{s}{(t-s)} \sup _{u \in \mathbb{R}^{3}}|\nabla \chi(u) \cdot u|,(\mathrm{C} .2)
\end{aligned}
$$

where $c$ is sufficiently large and in the first step we made use of Lemma 2.2 (a) and Lemma C.1 stated below.

The above proof relies on the following lemma: 
Lemma C.1. Let $\chi$ be a bounded operator on the single-photon space. Then

$$
\left\|\mathrm{d} \Gamma(g \chi g)\left(1+H_{\mathrm{ph}}\right)^{-1}\right\| \leq c\|\chi\|,
$$

where $c$ is independent of $\chi$ and $g \in C_{0}^{\infty}(\mathbb{R})_{\mathbb{R}}$ vanishes in a neighbourhood of zero.

Proof. Without loss of generality we can assume that $\chi$ is self-adjoint. We note the following

$$
\begin{aligned}
\mathrm{d} \Gamma(g \chi g)\left(1+H_{\mathrm{ph}}\right)^{-1} & =\left[\mathrm{d} \Gamma(g \chi g),\left(1+H_{\mathrm{ph}}\right)^{-1 / 2}\right]\left(1+H_{\mathrm{ph}}\right)^{-1 / 2} \\
+ & \left(1+H_{\mathrm{ph}}\right)^{-1 / 2} \mathrm{~d} \Gamma(g \chi g)\left(1+H_{\mathrm{ph}}\right)^{-1 / 2}
\end{aligned}
$$

The second term on the r.h.s. above satisfies

$$
\left(1+H_{\mathrm{ph}}\right)^{-1 / 2} \mathrm{~d} \Gamma(g \chi g)\left(1+H_{\mathrm{ph}}\right)^{-1 / 2} \leq\|\chi\|\left\|\omega(k)^{-1} g^{2}(k)\right\| H_{\mathrm{ph}}\left(1+H_{\mathrm{ph}}\right)^{-1} .
$$

As for the first term, we recall that

$$
\left(1+H_{\mathrm{ph}}\right)^{-1 / 2}=\frac{1}{\pi} \int_{0}^{\infty} d \lambda \lambda^{-\frac{1}{2}} \frac{1}{\lambda+1+H_{\mathrm{ph}}} .
$$

Thus we obtain

$$
\begin{aligned}
& i\left[\mathrm{~d} \Gamma(g \chi g),\left(1+H_{\mathrm{ph}}\right)^{-1 / 2}\right] \\
= & \frac{1}{\pi} \int_{0}^{\infty} d \lambda \lambda^{-\frac{1}{2}}\left(\lambda+1+H_{\mathrm{ph}}\right)^{-1} \mathrm{~d} \Gamma(g i[\chi, \widetilde{\omega}] g)\left(\lambda+1+H_{\mathrm{ph}}\right)^{-1} \\
\leq & \|[\chi, \widetilde{\omega}]\|\left\|\omega(k)^{-1} g^{2}(k)\right\| \frac{1}{\pi} \int_{0}^{\infty} d \lambda \lambda^{-\frac{1}{2}}\left(\lambda+1+H_{\mathrm{ph}}\right)^{-1} H_{\mathrm{ph}}\left(\lambda+1+H_{\mathrm{ph}}\right)^{-1} \\
\leq & \|[\chi, \widetilde{\omega}]\|\left\|\omega(k)^{-1} g^{2}(k)\right\| \frac{1}{\pi} \int_{0}^{\infty} d \lambda \lambda^{-\frac{1}{2}}(\lambda+1)^{-1}
\end{aligned}
$$

where $\widetilde{\omega}$ is a smooth, compactly supported function which coincides with $\omega$ on the support of $g$. Since the integral on the r.h.s. of (C.7) is convergent, the proof of the lemma is complete.

\section{References}

[1] A. Abdesselam and D. Hasler. Analyticity of the ground state energy for massless Nelson models. Commun. Math. Phys. 310, (2012) 511-536.

[2] H. Araki and R. Haag. Collision cross sections in terms of local observables. Commun. Math. Phys. 4, (1967) 77-91.

[3] W. Arveson. The harmonic analysis of automorphism groups. In Operator algebras and applications, Part I (Kingston, Ont., 1980), Proc. Sympos. Pure Math., 38, Amer. Math. Soc., Providence, R.I.,1982.D., pp. 199-269. 
[4] F. Bloch and A. Nordsieck. Note on the radiation field of the electron. Phys. Rev. 52, (1937) 54-59.

[5] D. Buchholz. Collision theory for massless bosons. Commun. Math. Phys. 52, (1977) 147-173.

[6] D. Buchholz. Gauss' law and the infraparticle problem. Phys. Lett. B 174, (1986) 331-334.

[7] D. Buchholz. Harmonic analysis of local operators. Commun. Math. Phys. 129, (1990) 631-641.

[8] D. Buchholz, M. Porrmann and U. Stein. Dirac versus Wigner: Towards a universal particle concept in local quantum field theory. Phys. Lett. B 267, (1991) 377-381.

[9] T. Chen, J. Faupin, J. Fröhlich and I.M. Segal. Local decay in non-relativistic QED. Commun. Math. Phys. 309, (2012) 543-582.

[10] T. Chen, J. Fröhlich and A. Pizzo. Infraparticle scattering states in nonrelativistic QED: I. The Bloch-Nordsieck paradigm. Commun. Math. Phys. 294, (2010) 761-825.

[11] T. Chen, J. Fröhlich and A. Pizzo. Infraparticle scattering states in nonrelativistic QED: II. Mass shell properties. J. Math. Phys. 50, (2009) 012103.

[12] V. Chung. Infrared divergence in quantum electrodynamics. Phys. Rev. 140B, (1965) 1110-1122.

[13] J. Dereziński and C. Gérard. Scattering theory of classical and quantum Nparticle systems. Springer 1997.

[14] J. Dereziński and C. Gérard. Asymptotic completeness in quantum field theory. Massive Pauli-Fierz Hamiltonians. Rev. Math. Phys. 11, (1999) 383-450.

[15] J. Dereziński and C. Gérard. Scattering theory of infrared divergent PauliFierz Hamiltonians. Ann. H. Poincaré 5, (2004) 523-577.

[16] W. De Roeck and A. Kupiainen. 'Return to equilibrium' for weakly coupled quantum systems: a simple polymer expansion. Commun. Math. Phys. 305, (2011) 797-826.

[17] W. Dybalski. Haag-Ruelle scattering theory in presence of massless particles. Lett. Math. Phys. 72, (2005) 27-38.

[18] W. Dybalski. Continuous spectrum of automorphism groups and the infraparticle problem. Commun. Math. Phys. 300, (2010) 273-299.

[19] W. Dybalski and Y. Tanimoto. Infraparticles with superselected direction of motion in two-dimensional conformal field theory. Commun. Math. Phys. 311, (2012) 457-490. 
[20] L. Faddeev and P. Kulish. Asymptotic conditions and infrared divergences in quantum electrodynamics. Theor. Math. Phys. 4, (1971) 745-757.

[21] J. Fröhlich. On the infrared problem in a model of scalar electrons and massless, scalar bosons. Ann. Inst. H. Poincaré Sect. A (N.S.) 19, (1973) 1-103.

[22] J. Fröhlich, M. Griesemer and B. Schlein. Asymptotic electromagnetic fields in models of quantum-mechanical matter interacting with the quantized radiation field. Adv. Math. 164, (2001) 349-398.

[23] J. Fröhlich, M. Griesemer and B. Schlein. Asymptotic completeness for Compton scattering. Commun. Math. Phys. 252, (2004) 415-476.

[24] M. Griesemer and H. Zenk. Asymptotic electromagnetic fields in nonrelativistic QED: The problem of existence revisited. J. Math. Anal. Appl. 354, (2009) 339-346.

[25] G.M. Graf. Asymptotic completeness for $N$-body short-range quantum systems: a new proof. Commun. Math. Phys. 132, (1990) 73-101.

[26] R. Haag. Local quantum physics. Second edition. Springer-Verlag, Berlin, 1996.

[27] R. Haag. Quantum field theories with composite particles and asymptotic conditions. Phys. Rev. 112, (1958) 669-673.

[28] D. Hasler and I. Herbst. Absence of ground states for a class of translation invariant models of non-relativistic QED. Commun. Math. Phys. 279, (2008) 769-787.

[29] J.M. Jauch and F. Rohrlich. The infra-red divergence. Helv. Phys. Acta 27, (1954) 613-636.

[30] K. Johannsen. Teilchenaspekte im Schroermodell. Diplomarbeit, Universität Hamburg 1991.

[31] T. Kibble. Coherent soft-photon states and infrared divergencies. I. Classical currents. J. Math. Phys. 9, (1968) 315.

[32] A. Pizzo. One-particle (improper) states in Nelson's massless model. Ann. Henri Poincaré 4, (2003) 439-486.

[33] A. Pizzo. Scattering of an infraparticle: the one particle sector in Nelson's massless models. Ann. Henri Poincaré 6, (2005) 553-606.

[34] M. Porrmann. Particle weights and their disintegration I. Commun. Math. Phys. 248, (2004) 269-304.

[35] M. Porrmann. Particle weights and their disintegration II. Commun. Math. Phys. 248, (2004) 305-333. 
[36] M. Reed and B. Simon. Methods of modern mathematical physics I. Functional analysis. Academic Press, 1972.

[37] D. Ruelle. On the asymptotic condition in quantum field theory. Helv. Phys. Acta 35, (1962) 147-163.

[38] U. Stein. Zur Konstruktion von Streuzuständen mit Hilfe lokaler Observabler. Ph.D. Thesis, Universität Hamburg 1989.

[39] R.F. Streater and A. Wightman. PCT, spin and statistic and all that. Princeton University Press 1980.

[40] D.R. Yennie, S.C. Frautschi and H. Suura. The infrared divergence phenomena and high-energy processes. Annals of Physics 13, (1961) 379-452. 